\title{
Wilson-Loop Symmetry Breaking Reexamined
}

\author{
Atsushi Nakamula \\ Department of Physics, Tokyo Metropolitan University, \\ Setagaya-ku, Tokyo 158, Japan \\ and \\ Kiyoshi Shiraishi \\ Institute for Nuclear Study, University of Tokyo, \\ Midori-cho, Tanashi, Tokyo 188, Japan
}

Phys. Lett. B215, No. 3 (1988) 551; B218, No. 4 (1989) 508 (E).

\begin{abstract}
The splitting in energy of gauge field vacua on the non-simply connected space $S^{3} / Z_{2}$ is reconsidered. We show the calculation to the oneloop level for a Yang-Mills vector with a ghost field. We confirm our previous result and give a solution to the question posed by Freire, Romão and Barroso.
\end{abstract}

The origin of the gauge symmetry group, which includes that of the standard model, is still unclear. Unified theories of fundamental forces assume a large symmetry group which is to be broken at the low-energy scale.

Recently developments of string theories [1] originated from the fact that the gauge group is uniquely determined due to quantum-mechanical consistency [2]. The theories are formulated in higher dimensions. In more recent days, a large number of "four-dimensional string models" [3] with various symmetry groups were constructed at the sacrifice of uniqueness; the "right" choice of gauge group for string theory is still unknown, however [4].

There is the interesting possibility that compactification and gauge symmetry breaking take place altogether in higher-dimensional theory with the gauge theory of a large group. The mechanism dubbed the Wilson-loop mechanism has been used in the context of gauge symmetry breaking in superstring theories $[5]$.

But the phenomenon can be completely explained by means of the fieldtheoretical concept. Indeed, simple examples with an extra one-dimensional compact space, a circle, were provided by Hosotani [6]. He also pointed out the importance of the calculation of a Casimir-like energy, in order to choose a "correct" vacuum; namely, we have to look for the gauge vacuum which has the lowest energy.

A more complex model was first considered by Evans and Ovrut [7]. The model is formulated in the spacetime $M_{4} \times S^{3} / Z_{2}$, where $M_{4}$ denotes flat four- 
dimensional spacetime and $S^{3} / Z_{2}$ means a three-sphere on which antipodal points are identified. They, however, did not give an explicit computation of the value of vacuum energies.

One of the present authors carried out [8] an evaluation of the one-loop vacuum energy difference by a sort of dimensional regularization a la Candelas and Weinberg [9]. Then he concluded that the lower energy vacuum is the vacuum which realizes the unbroken gauge symmetry.

Recently, however, the authors of ref. [10] claim that the vacuum of broken gauge symmetry has a lower energy than that of unbroken symmetry.

We examine in this letter the one-loop calculation of the energy difference of two vacua which arise in the model on the spacetime $M_{4} \times S^{3} / Z_{2}$, and give a solution to the question. Most of our results were published in ref. [8]. We carefully confirm the results as one will see.

Let the metric on $S^{3}$ be of the form

$$
d s^{2}=r^{2}\left[d \psi^{2}+\sin ^{2} \psi\left(d^{2} \theta+\sin ^{2} \theta d \phi^{2}\right)\right],
$$

where $r$ is the radius of $S^{3}$. A $Z_{2}$ transformation is defined by

$$
\psi \rightarrow \pi-\psi, \quad \theta \rightarrow \pi-\theta, \quad \phi \rightarrow \phi+\pi
$$

If we pick out invariant states or harmonics under this transformation, identification of the antipodal points on $S^{3}$ is attained $[7,8,10]$.

Throughout this letter, the background spacetime is fixed (by hand). Furthermore, in our terminology, the words "energy" and "energy density" are used with no strict distinction. There appears no ambiguity or confusion as long as we consider a static, fixed spacetime.

As a simple, specific model, and for the sake of comparison, we suppose an $S U(3)$ gauge field only, although the choice of gauge group is irrelevant to the conclusion, as we will see.

Here, capital Latin indices have the range $M, N=0, \ldots, 6$; Greek indices $\mu, \nu$ run over $0,1,2,3$ and lowercase Latin indices $m, n$ run over $4,5,6$. Thus the $S U(3)$ gauge field is written as

$$
A_{M}^{a}=\left(A_{\mu}^{a}, A_{m}^{a}\right)
$$

where $a$ is the group index of the adjoint representation. We have to note, first of all, that the classical configurations of the gauge field allowed on $S^{3} / Z_{2}$ are not only trivial ones like

$$
A_{m}^{a(1)}=(0,0,0)
$$

but also, for example [7],

$$
A_{m}^{a(2)}=\left(0,0,2 \sqrt{3} \delta^{a 8}\right) .
$$

or [10]

$$
A_{m}^{a(3)}=\left(0,0,2 \delta^{a 3}\right),
$$


to satisfy $F_{m n}^{a}=D_{m} A_{n}^{a}-D_{n} A_{m}^{a}+f^{a b c} A_{m}^{b} A_{n}^{c}=0$, where $D_{m}$ is the covariant derivative and $f^{a b c}$ is the structure constant of the group. These configurations of which the field strengths vanish are called vacuum gauge fields. The above gauge configurations have $Z_{2}$ invariance defined on $S^{3}$. The configurations $A_{m}^{a(1)}$ and $A_{m}^{a(2)}$ are inequivalent modulo a proper gauge transformation on $S^{3} / Z_{2}$; but $A_{m}^{a(2)}$ and $A_{m}^{a(3)}$ are connected by a global group rotation. Thus the vacuum associated with $A_{m}^{a}{ }^{(2)}$ and that of $A_{m}^{a}{ }^{(3)}$ are identical. In phenomenology one can think of the vacuum gauge field as a configuration of adjoint Higgs fields.

Now, we are going to calculate the energy difference between the different gauge vacua. To compute the one-loop vacuum energy, we only need to know eigenstates and eigenvalues of Laplacians which function on the quantum fluctuation of the fields [9]. The eigenstates of Laplacians defined on $S^{3}$ are classified even or odd under the $Z_{2}$ transformation (2) $[7,8,10]$. Namely, the harmonics of the even modes are invariant under the $Z_{2}$ transformation while those of the odd modes change sign under the $Z_{2}$ transformation.

In the trivial vacuum $\left(A_{m}^{a(1)}\right)$ on $S^{3} / Z_{2}$ all states are even modes, on the other hand, in the nontrivial vacua $\left(A_{m}^{a}{ }^{(2)}\right)$ on $S^{3} / Z_{2}$ the states corresponding to the component which do not commute with the vacuum gauge fields are odd modes because of their coupling to the vacuum field; the other states corresponding to the commuting component remain to be even modes. In general, the eigenvalues and eigenfunctions of Laplacians on $S^{N}$ ( $N$-sphere) for various tensor fields are well known [11] and "even" and "odd" modes are apparently distinguished from each other by a quantum number which also directly determines the eigenvalue $[7,8,10]$.

Of course, the zero mode of the gauge field belongs to the even modes. Thus the gauge symmetry is reduced to be small in nontrivial vacua since the odd modes involve massive excitations only.

Obviously, the total difference in energy is proportional to the difference in energy for a single degree of freedom; we do not need group theoretical complexity but need only a naive counting of the number of group indices attached to the components that are noncommuting with the vacuum gauge fields (after diagonalization, if necessary).

Let us denote the energy (density) of each vacuum as $E^{(1)}$ and $E^{(2)}$. Further, we define the energy difference for a single degree of the group index, $\Delta E$, which is the difference in the vacuum energy obtained from the one-loop calculation adopting even and odd modes on $S^{3}[7,8,10]$ (see further on). Because it is $Z_{2}$ that classifies the harmonics on $S^{3}$, the energy difference between any vacua is determined by the single quantity $\Delta E$. The difference in energy is expressed as

$$
E^{(1)}-E^{(2)}=4 \Delta E
$$

because the vacuum of the gauge configuration $A_{m}^{(2)}$ realizes four massless gauge bosons for $S U(2) \times U(1)$. Thus the sign of $\Delta E$ is only relevant to the choice of the lower energy vacuum, that is to say, whether gauge symmetry breaking occurs or not. 
Next we will give the derivation of $\Delta E$.

A higher dimensional vector field contains many kinds of fields, or harmonics, from the point of view of four dimensions after dimensional reduction. The most naive classification divides $A_{M}^{a}$ into $A_{\mu}^{a}$, and $A_{m}^{a}$. The eigenfunctions of the Laplacian for $A_{\mu}^{a}$ must contain scalar harmonics on $S^{3} / Z_{2}$, in the usual sense of Kaluza-Klein theory. Thus the eigenvalue is the same as that of a scalar field on $S^{3} / Z_{2}$ and the degeneracy is four times that since the flat spacetime index runs from 0 to 3 .

The eigenfunctions for $A_{m}^{a}$ have already been investigated [11]. There are two kinds of eigenfunctions. One is the transverse part, the other is the longitudinal part; the latter is known to be expressed as a covariant derivative of the scalar harmonics on $S^{3}$ [11]; and the eigenvalues of the longitudinal vector including the contribution of the Ricci curvature tensor also coincide with the ones of the scalar harmonics. The even and odd modes under $Z_{2}$ transformation for the longitudinal vector harmonics are similarly defined as the scalar case.

Finally, we consider a ghost field. A complex ghost field for vector fields has two scalar degrees of freedom and a negative contribution to the one-loop energy.

We define the energy differences per degree of freedom for a scalar, a transverse vector, a longitudinal vector on $S^{3} / Z_{2}$, a complex ghost and a fourdimensional vector as $\Delta E_{S}, \Delta E_{T V}, \Delta E_{L V}, \Delta E_{G}$ and $\Delta E\left(A_{\mu}^{a}\right)$. For example, $\Delta E$ is formally expressed as an infinite sum a la Candelas and Weinberg [9];

$$
\Delta E_{S}=\lim _{n \rightarrow 4}\left(-\frac{1}{2(4 \pi)^{n / 2} r^{n}} \Gamma\left(-\frac{n}{2}\right) \sum_{l=0}^{\infty}(-1)^{l}(l+1)^{2}[l(l+2)]^{n / 2}\right)
$$

This is just the difference between the even and odd sectors of the spectra on $S^{3}[8,10]$. Each sector corresponds to even $l$ and odd $l$ respectively. The other $\Delta E$ 's can be written in the same manner [12].

From the above consideration, there are the following relations among the $\Delta E$ 's:

$$
\Delta E_{L V}=\Delta E_{S}, \quad \Delta E_{G}=-2 \Delta E_{S}, \quad \Delta E\left(A_{\mu}^{a}\right)=4 \Delta E_{S} .
$$

The total energy difference $\Delta E$ for a vector field in $M_{4} \times S^{3} / Z_{2}$ is written as

$$
\begin{aligned}
\Delta E & =\Delta E\left(A_{\mu}^{a}\right)+\Delta E_{T V}+\Delta E_{L V}+\Delta E_{G} \\
& =4 \Delta E_{S}+\Delta E_{T V}+\Delta E_{S}-2 \Delta E_{S} \\
& =\Delta E_{T V}+3 \Delta E_{S} .
\end{aligned}
$$

The authors of ref. [11] calculated $\Delta E_{T V}$ and $\Delta E_{G}$, however they seemed to forget not only the longitudinal vector but also the contribution from a fourdimensional vector. The values of $\Delta E_{T V}$ and $\Delta E_{S}$ can be read from ref. [8], they are

$$
\begin{aligned}
& \Delta E_{T V}=6.312 \times 10^{-3} r^{-4}, \\
& \Delta E_{S}=-5.116 \times 10^{-3} r^{-4} .
\end{aligned}
$$


Accordingly $\Delta E$ [eq. (10)] is negative, thus an unbroken gauge vacuum is favorable. This conclusion is also applicable to any gauge group as long as we consider $S^{3} / Z_{2}$.

The authors of ref. [7] omitted a concrete calculation o n $M_{4} \times S^{3} / Z_{2}$. The authors of ref. [11] drew the wrong conclusion because they forgot to consider the contribution of the four-dimensional vectors $A_{\mu}^{a}$. The confirmation of the counting of degrees of freedom can be done by computing the free energy that concerns each vacuum by means of the introduction of temperature.

We now consider the one-loop correction to the "potential" obtained in ref. [13]. The authors of ref. [13] discovered the path that connects two gauge vacua. We would like to consider the loop correction to the potential in order to study the (quasi-) stable domain wall structure at zero and finite temperature $[12]$.

K.S. is grateful to the Japan Society for the Promotion of Science for a fellowship. He also thanks Iwanami Fūjukai for financial aid.

The author would like to thank J.S. Dowker and S. P. Jadhav for pointing out the error in the paper.

\section{References}

[1] M.B. Green, J.H. Schwarz and E. Witten, Superstring theory, Vols. 1,2 (Cambridge U.P., Cambridge, 1987).

[2] M.B. Green and J.H. Schwarz, Phys. Left. B149 (1984) 117.

[3] H. Kawai, D.C. Lewellen and S.-H. H. Tye, Nucl. Phys. B288 (1987) 1; W. Lerche, D. Lüst and A. N. Schellekens, Nucl. Phys. B287 (1987) 477; I. Antoniadis and C. P. Bacbas, Nucl. Phys. B289 (1987) 87.

[4] L.J. Dixon, V.S. Kaplunovsky and C. Vafa, Nucl. Phys. B294 (1986) 43; M. Kato, T. Koikawa and M. Tatewaki-Hatsuda, Mod. Phys. Lett. A3 (1988) 1603.

[5] E. Witten, Nucl. Phys. B258 (1985) 75; J. Breit, B. Ovrut and G. Segré, Phys. Lett. B158 (1985) 33; A. Sen, Phys. Rev. Lett. 55 (1985) 33; K. Choi and J. E. Kim, Phys. Lett. B176 (1986) 103.

[6] Y. Hosotani, Phys. Lett. B126 (1983) 309.

[7] M. Evans and B. Ovrut, Phys. Lett. B174 (1986) 63; B. A. Ovrut, Prog. Theor. Phys. Suppl. 86 (1986) 185.

[8] K. Shiraishi, Prog. Theor. Phys. 78 (1987) 535.

[9] P. Candelas and S. Weinberg, Nucl. Phys. B237 (1984) 397.

[10] F. Freire, J.C. Romeo and A. Barroso, Phys. Lett. B206 (1988) 491. 
[11] M. A. Rubin and C.R. Ordoñez, J. Math. Phys. 25 (1984) 2888; 26 (1985) 65.

[12] A. Nakamula and K. Shiraishi, in preparation.

[13] K. Lee, R. Holman and E.W. Kolb, Phys. Rev. Lett. 59 (1987) 1069. 